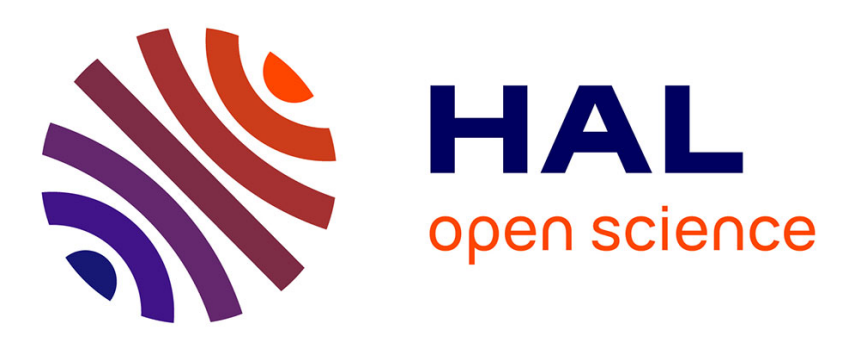

\title{
Cochlear Implants: Consequences of Microphone Aging on Speech Recognition
}

\author{
Christian Berger-Vachon, Pierre Antoine Cucis, Eric Truy, Hung Thai Van, \\ Stéphane Gallego
}

\section{To cite this version:}

Christian Berger-Vachon, Pierre Antoine Cucis, Eric Truy, Hung Thai Van, Stéphane Gallego. Cochlear Implants: Consequences of Microphone Aging on Speech Recognition. Berger-Vachon C., Gil Lafuente A., Kacprzyk J., Kondratenko Y., Merigó J., Morabito C. Complex Systems: Solutions and Challenges in Economics, Management and Engineering., Springer International Publishing, pp. 497-519, 2018, Studies in systems, Decision and control, 978-3-319-69989-9. 10.1007/978-3-319-699899_30.hal-01667763

\section{HAL Id: hal-01667763 https://hal.science/hal-01667763}

Submitted on 19 Dec 2017

HAL is a multi-disciplinary open access archive for the deposit and dissemination of scientific research documents, whether they are published or not. The documents may come from teaching and research institutions in France or abroad, or from public or private research centers.
L'archive ouverte pluridisciplinaire HAL, est destinée au dépôt et à la diffusion de documents scientifiques de niveau recherche, publiés ou non, émanant des établissements d'enseignement et de recherche français ou étrangers, des laboratoires publics ou privés. 


\title{
COCHLEAR IMPLANTS: CONSEQUENCES OF MICROPHONE AGING ON SPEECH RECOGNITION
}

\author{
C. Berger-Vachon ${ }^{1,2,3}$, P.A. Cucis ${ }^{1}$, E. Truy ${ }^{1,2,5}$, H. Thai Van ${ }^{1,2,5}$, S. Gallego ${ }^{1,4}$ \\ ${ }^{1}$ University Claude-Bernard Lyon1, 43 bd du 11 Novembre, 69622 Villeurbanne-Cedex, France \\ ${ }^{2}$ Lyon Neurosciences Research Centre, (CRNL), INSERM U1028, 95 bd Pinel, 69675 Bron-Cedex, France \\ ${ }^{3}$ LBMC-IFSTTAR, 25 Avenue François Mitterrand, 69500 Bron \\ ${ }^{4}$ Audition-Conseil Centre, 34 avenue Lacassagne, 69003 Lyon, France \\ ${ }^{5}$ CRIC, ORL Building, Edouard-Herriot Hospital, place d'Arsonval69437 Lyon-Cedex 03, France
}

\section{ABSTRACT}

Aging is a general phenomenon which affects everything and everybody in this world. Designed for the rehabilitation of profound deafness, cochlear implants $(\mathrm{Cl})$ do not escape to this general rule. One very insidious effect concerns the microphone as an ongoing drift occurs over the time. In this work we wish to assess the consequences of this evolution on speech recognition.

In order to perform this task, a general population of $\mathrm{Cl}$ users and $\mathrm{NH}$ subjects (using a $\mathrm{Cl}$ simulator) participated to this study. They listened to French dissyllabic words and we recorded recognition percentages. Words were presented to the listeners in noise with a variable signal to noise ratio (SNR) and the percentages ranged from $0 \%$ to $100 \%$.

For the $\mathrm{Cl}$ simulator, the drift was simulated from data coming from figures measured on regular hearing aids. This choice seems relevant as $\mathrm{Cls}$ and hearing aids use the same microphones. Also, the $\mathrm{Cl}$ simulator we used, picked up the general principles of a vocoder to represent the classical coding strategies used in Cls ("CIS-like" and n-of-m).

With $\mathrm{Cl}$ users, the results were compared before and after cleaning the microphones; also, in a subgroup of $\mathrm{Cl}$ users, we performed the replacement of the head filter protecting the microphone and the recognition percentages were compared with those coming from the standard "Brush and Blow" cleaning procedure. The results have been revisited and quantified after a curve fitting.

The outcomes indicated that the "CIS-like" coding schemes were less sensitive to aging than the n-of-m strategies. Also, cleaning ameliorated the recognition performances, but the increase was not dramatically high. Furthermore, the improvement mainly occurred in the middle of the SNR range where the noise was not too intense. We made these observations with $\mathrm{Cl}$ users and with $\mathrm{NH}$ subjects indicated that the results should be linked to the properties of the signal.

Finally, as we cannot stop the consequences of aging, we can set up an action plan to reduce its effect. And this is true in everyday life. In the case of $\mathrm{Cls}$, a lot of solutions are available, among them the choice of the sound coding strategy and the periodicity of the clinical check and device setting.

KEY WORDS: Cochlear Implants, Microphone aging, Cl \& NH listeners, Coding strategies, Cleaning procedures, Syllable recognition in noise. 


\section{COCHLEAR IMPLANTS AND AGING}

Cochlear implants (CI) have successfully restored partial hearing to over 400,000 deaf persons in the world and the rhythm of implantations is high in the world with a minimal rate of about 30,000 implantations [1].

Economically, $\mathrm{Cl}$ is a good investment for the society. The machine is expensive (about 25,000 \$), the surgery also and the follow up of the patient during the first year (speech therapy, audiology) pushes this figure to $50,000 \$$. Nevertheless, if we consider the cost of a handicapped persons during his life (education in dedicated centers, perturbed familial life, professional limitations and so on) fitting a deaf person with a $\mathrm{Cl}$ is worthwhile to be done and many social systems cover the expense mostly when it is for children.

\section{Reliability}

Designed to last for more than ten years, $\mathrm{Cl}$ suffers from failures and some of them come from aging $[2,3]$ and one of the goal of engineering is to reduce their impact. In this paper we wish to discuss this issue.

Cochlear implants are biomedical prostheses which restore some hearing in profoundly deaf subjects. Basically, they are made with two main components, an external device which is for speech reception and processing, and an implant which decodes the information transmitted by the external device and distributes it to the ends of the auditory nerve in the inner ear. All the elements are subjected to aging and patients are deeply affected when failures occur in the system. There are two kinds of failure, the hard failure easy to detect as the machine breakdown is seen immediately and the soft failure when the failure is intermittent. Another kind of burden, more insidious, is the progressive loss of efficiency on one of the components of the machine. Among them the attack of the microphone is one of the hazards affecting the machine. Several alerts have been raised and some dramatic stories about alterations on the opening in hearing aids have issued warnings [4] which must not be ignored. A check of the microphone entrance often discovers debris accumulated in undesirable locations and the input sound pathway is more or less obliterated.

\section{Microphone aspect}

The microphone repair issue is well ranked into the external failures of a cochlear implant $[5,6]$. It comes in the fourth or fifth position in the classification after the headpiece (antenna), the battery compartment and the speech processor and it represents nearly $11 \%$ of the repairs.

But the progressive drift of the microphone properties is often not noticed and everybody should keep in mind that the microphone works in very adverse environments which can range from very cold and dry to very humid and hot, with a common exposure to sweat, dirt, dust, grease and so on. This is a very aggressive situation and moisture and grease are likely to affect the sensitivity of the microphone and its frequency response. 
Consequently this behavior should be studied and this aspect of aging should not be ignored. In a previous approach Razza et al $[7,8]$ indicated that speech tracking scores are correlated significantly with the loss of the microphone sensitivity. Responses curves were compared to those of fully working microphones and the consequences on speech recognition need to be studied.

This situation is not very simple as the use of cochlear implants occurs often in noise which is part of our usual environment.

\section{Coding strategies}

$\mathrm{Cl}$ allows several coding strategies which can be implanted on almost all the machines, and the choice of a relevant coding strategy is something which must be discussed.

Basically, in $\mathrm{Cl}$, the speech input spectrum is divided into frequency bands, and this "simplified" spectrum is distributed to the electrodes distributed along the cochlea which is the hearing organism.

Two main schemes are usually taken to program the speech processors by the $\mathrm{Cl}$ manufacturers:

- $\mathrm{n}$-of-m: the speech spectrum is divided into $\mathrm{m}$ bands, and the $\mathrm{n}$ with the highest energy are kept and transmitted to the cochlea. This scheme is popular, because the presentation of selected acoustic features corresponds to an approach based on the phonetic theory. In this circumstance, only a subset of the available electrodes is stimulated. Also, as the number of active electrodes is limited, it is expected that the overlap between two successive stimulations is small, but this may be not true if two consecutive electrodes are selected [9].

- CIS (Continuous Interleaved Sampling): it is a sound processing scheme designed to enhance the delivery of temporal fine structure to the implant, in order to beat the poor intelligibility of speech in a competing noise. All the band pass filters are kept and the number of electrodes is reduced, leading to a wide spacing, between two consecutive electrodes, which is likely to reduce the interaction. Also, the stimulation rate is high and it enhances the delivery of temporal fine structure to the implant, and this is an advantage in a competing noise [10].

\section{Noise influence}

The choice of the noise is also a major issue. The effect of listening conditions determines the performances of the listeners. The signal to noise ratio (SNR) is an important parameter for the assessment of the recognition performances. Lowering the SNR spoils the incoming speech signal and this action is worthy to be measured. Also, with different values of the SNR it is easy to avoid the ceiling and the floor effects, enabling the determination of some characteristic parameters traditionally used in the evaluation of speech recognition [11].

In our work, the choice of the Fournier's lists (a French equivalent to the spondee lists) led to recognition percentages ranging from $0 \%$ to $100 \%$, according to the SNR, and it was suitable to the aims of our experiment.

\section{$\mathrm{NH}$ versus $\mathrm{Cl}$}


Two aspects are generally considered when coding strategies are examined in cochlear implants: the recognition seen with patients using the coding schemes, and the behavior of normal hearing (NH) subjects facing the signal (a simulation is done for $\mathrm{NH}$ subjects). Both performances are complementary as in one case ( $\mathrm{Cl}$ users) we have the direct results with the group of persons concerned by the prosthesis and in the second group we can test the signal action using a more comprehensive approach [12]. In other words, one matter is more oriented to the patient and the other tests the signal. Both groups receive the same amount of information about speech, but the outcomes may be different or similar [13,14]. Furthermore, the $\mathrm{Cl}$ users group is very heterogeneous and the standard deviation in the measures is large making the statistical interpretation of the results more difficult. On the contrary, with the $\mathrm{NH}$ listeners, several strategies and microphone drift are evaluated on the same people and intra-subject studies can be done making the evaluation more accurate.

\section{Organization of this study}

The aim of this study is to assess the behavior of two coding strategies (n-of-m and CIS) in front of the aging of the $\mathrm{Cl}$ microphone, and to give some indication to the audiologists in charge of $\mathrm{Cl}$ settings in the specialized clinical centers.

After an introduction of the $\mathrm{Cl}$ issues about aging and the main elements which will be manipulated to carry out the study, the second section introduces the tools for the simulation $(\mathrm{Cl}$ and microphone drift), the subjects who participated to the experiment and the mathematical fitting of the recognition results. Then in the third section, the results (recognition percentages and audiological features) obtained with the $\mathrm{Cl}$ users and the $\mathrm{NH}$ subjects are presented.

The fourth section is a discussion about the different elements which were used in this work, The comparison of the performances of $\mathrm{Cl}$ users and $\mathrm{NH}$ subjects, the choice of the noise and evaluation of the strategy and how to reduce the influence of the microphone drift with aging. Finally, a conclusion sums up the main contributions of this work.

\section{MATERIAL AND METHODS}

\subsection{Stimulation}

\subsubsection{Cochlear implant principles}

We will not expose the details of signal processing in cochlear implants here, but only the essential principles (figure 1). Cochlear implant can be divided into two main parts:

- The external block (signal processor) processes the signal and separates the sound in multiple frequency bands (channels).

- The internal block (the implant) receives the information from the processor and distributes the energy of the channels to the electrodes along the cochlea.

The information is transmitted from the processor to the internal part through the skin by a radiofrequency signal. 
Sounds in the environment are captured by the microphones that are very impacted by soiling and aging. Then the processor fits the signal received from the microphones to the patient's physiology and separates the signal into multiple frequency bands. The frequency bands correspond to the treatment channels scaled using a logarithmic scale. We chose to use the Bark scale for our $\mathrm{Cl}$ simulator [15].

Most of the existing sound coding strategies are based on two main principles: there are "CIS-like" strategies and " $n$-of-m" strategies. It is interesting to notice that, for the n-of-m strategies, if $n=m$ it is very similar to $\mathrm{CIS}$. So the value of $\mathrm{n}$ is important to be considered.

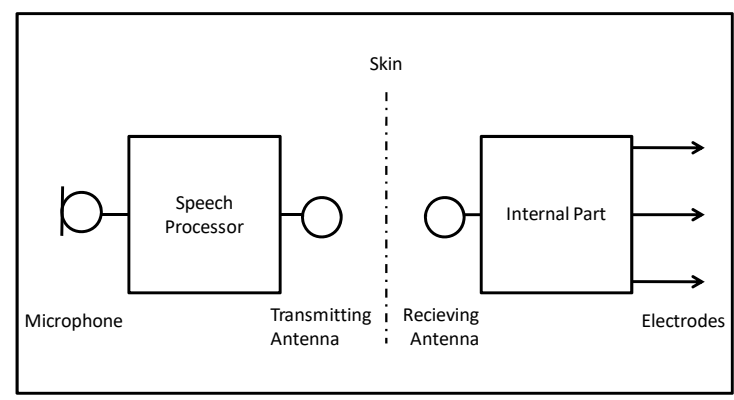

Figure 1: Shematic view of a cochlear implant

\subsection{2. $\underline{\text { Vocoder }}$}

The $\mathrm{Cl}$ simulator used for testing $\mathrm{NH}$ subjects is a basic vocoder fitted for the purpose of the study (figure 2).

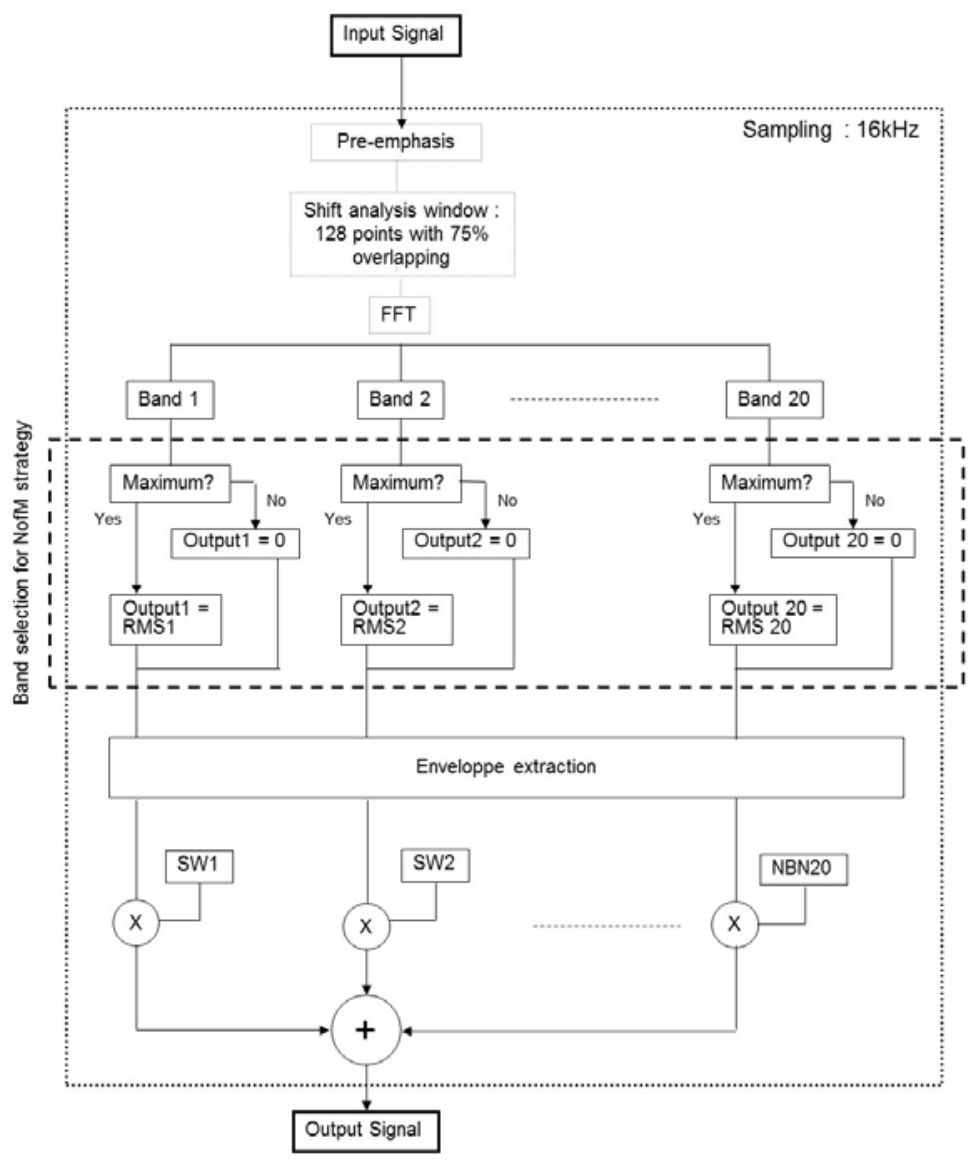

Figure 2: Block diagram representing the signal processing performed by the $n$-of-m simulator. 
The different steps of the signal processing are:

- The input signal goes through a pre-emphasis filter which is a high-pass filter (cutoff frequency $1.2 \mathrm{kHz}$ and slope $3 \mathrm{~dB}$ /octave).

- The signal is then sampled (16 kHz sampling frequency, 16-bit quantization), a short term fast Fourier transform (FFT) is applied to the samples, frame length is 128 points ( $8 \mathrm{~ms}$ ), leading to 64 spectral beams (amplitude and phase), ranging from 0 to $8 \mathrm{kHz}$. The step between two beams is $125 \mathrm{~Hz}$.

- Then, the spectral beams are grouped into frequency bands which are logarithmically distributed, according to the ear physiology (Bark scale). Considering the usual values taken in cochlear implants we used 20 bands (leading to 20 channels).

- In each band, the energy is calculated using the Parseval's formula (the squares of the amplitude of each beam are added). In the "CIS-like" coding all the channels are taken. For the $n$-of-m coding only the 8 more energetic channels are kept. The value $n=8$ is classical in cochlear implants. There is a frame overlap of 6 milliseconds ( $75 \%$ overlap), and a set of pulses is calculated for each frame.

- Each channel is represented by a narrow-band spectrum coming from a white noise spectrum. The amplitude of the narrow band follows the energy detected in the corresponding channel. The first two-channels, which are very narrow, were represented by sine waves. The output signal is obtained by summing the selected channels ( 8 for $n$-of-m; 20 for "CIS-like").

\subsubsection{Evaluation of the frequency drift of a hearing aid microphone}

In this study we tested speech recognition in noise with $\mathrm{Cl}$ users, before and after a microphone cleaning process. For $\mathrm{NH}$ subjects we used a $\mathrm{Cl}$ simulator integrating a low-pass filter mimicking the microphones frequency drift due to soiling. The low-pass filter approximated a measured median attenuation. We based our work on a study about microphone frequency drift due to soiling on conventional hearing-aids. Knowles electronics (Itasca, Illinois, USA) is the manufacturer which leads the market of microphones for hearing-aids and cochlear implants. That is why we considered that this study matched for cochlear implants' microphones too.

The study of the microphones sensitivity loss had been conducted, during a routine maintenance appointment in a hearing care center, on regular hearing aids worn by $\mathrm{n}$ hearing impaired patients ( $\mathrm{n}$ = 129) [16]. Two steps were necessary in order to check the microphone frequency drift. First the earphone and the ear mold were deeply cleaned leading to the state "clean earphone and dirty microphone". Secondly, the microphone was carefully cleaned, leading to the state "clean earphone and clean microphone". The subtraction of the two states gave the attenuation due to the microphone soiling. 
Then the microphone transfer function was evaluated after a frequency warble sweep ranging from $200 \mathrm{~Hz}$ to $8,000 \mathrm{~Hz}$ (at $60 \mathrm{~dB} \mathrm{SPL}$ ).

Four degrees of attenuation have been defined:

-Case 1: clean microphone (no loss); the response was not modified,

-Case 2: medium loss corresponding to the attenuation seen on $50 \%$ of the microphones (percentile 50\%)

-Case 3: strong loss, corresponding to the $20 \%$ worse microphones (percentile $80 \%$ ),

-Case 4: very strong loss, corresponding to the $10 \%$ worse microphones (percentile $90 \%$ ).

The clean signal was modified by the different attenuation curves and then passed through the vocoder.

On figure 3 , the three degrees of loss of sensitivity, corresponding to the $50 \%, 80 \%$ and $90 \%$ percentiles, are indicated. Each degree was represented by two straight lines for simplification. The signal attenuation started at $800 \mathrm{~Hz}$ and then three slopes were introduced, respectively, $2 \mathrm{~dB}, 7 \mathrm{~dB}$ and $9 \mathrm{~dB}$ per decade.

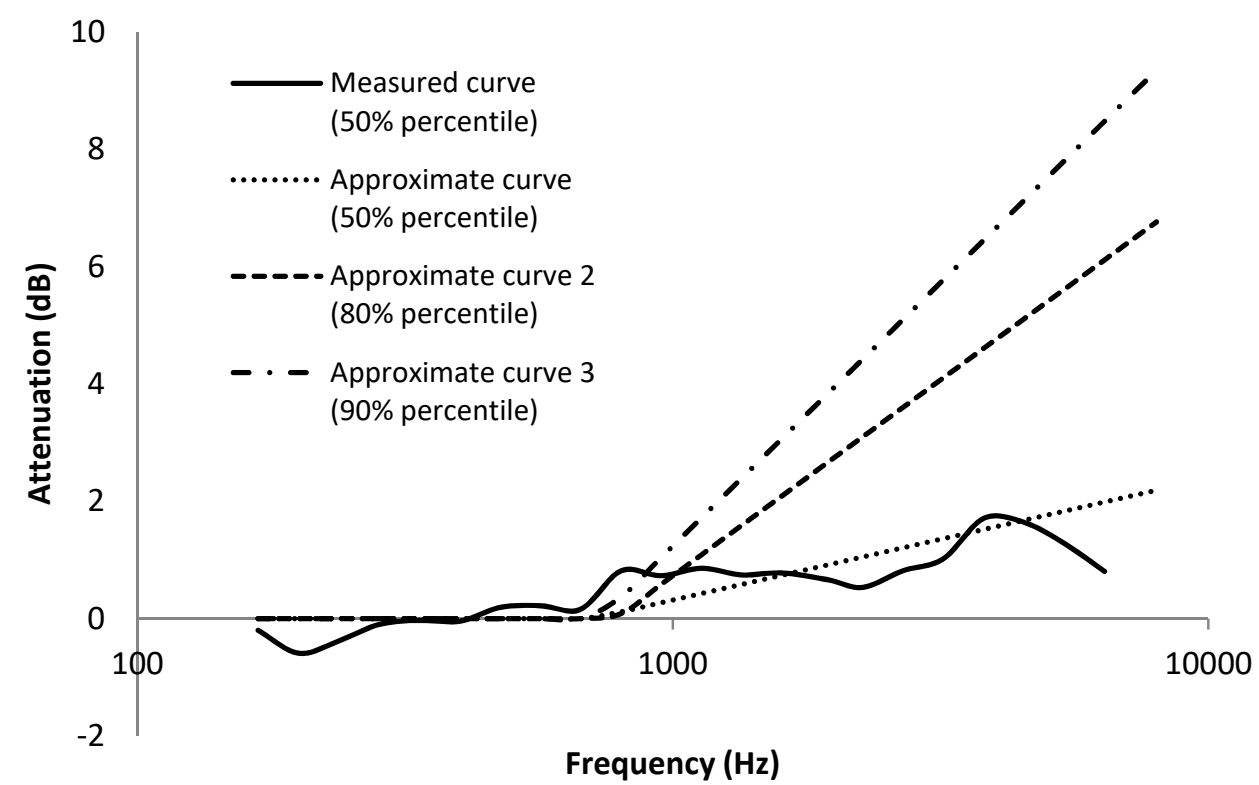

Figure 3 : Simulation of the microphone loss of sensitivity.

\subsection{Tests}

\subsubsection{Participants}

The work presented in this paper follows a previous pilot study [17] and it was approved by the French Ethics Committee "Sud Est 2" (August, 27, 2014), under the supervision of the HCL (Civil Hospices of Lyon). All the participants filled an agreement form prior entering the study and all the recordings were made by an audiologist. 


\subsubsection{1. $\quad \mathrm{Cl}$ users}

Sixty-one $\mathrm{Cl}$ patients were included in this study. Their ages ranged from 18 to 60 years (average 37 years old). Nineteen subjects were fitted with "CIS like" strategies and 42 had the n-of-m strategies.

The population of the $\mathrm{Cl}$ users had been parted into four groups:

-Subpopulation 1 (SP1): Med-El and Advanced-Bionics subjects (19 patients): "brush and blow" cleaning procedure and CIS-like coding strategies,

-Subpopulation 2 (SP2): Neurelec subjects (13 patients); "brush and blow" cleaning and n-ofm strategies,

-Subpopulation 3 (SP3): Cochlear" subjects (14 patients): "brush and blow" cleaning and n-ofm strategies,

-Subpopulation 4 (SP4): Cochlear ${ }^{\circ}$ subjects (15 subjects): replacement of the protective filter and n-of-m strategies.

\subsubsection{NH subjects}

Twenty $\mathrm{NH}$ subjects participated to this experiment. Their age ranged from 18 to 33 years (average 25 years). All the subjects had an ORL examination before entering the study, in order to eliminate previous pathologies or deafness which may corrupt the results. The auditory thresholds were measured; they were always below $20 \mathrm{~dB} \mathrm{HL}$ for all the frequencies between 250 and $8,000 \mathrm{~Hz}$. According to the BIAP (International Bureau for Audio-Phonology) all the subjects were considered as $\mathrm{NH}$ listeners.

\subsubsection{Session}

\subsubsection{Material}

In this work we used the French dissyllabic Fournier's lists. These lists were well adapted to the patients' recognition ability in this environment. They were uttered by a male voice and were the vocal part of the signal. They are similar to the spondees lists used in English. Each list contains 10 disyllabic words (for instance "le bouchon" = the cork). Forty lists are available, and the recognition unit was the syllable. So the recognition step was $5 \%$.

A cocktail-party noise has been used in this study. This noise was a mix of voices from 8 speakers, 4 males and 4 females.

\subsubsection{Test sessions}

The signal delivered to the subjects was made of the Fournier's lists mixed with the cocktail-party noise. The mixing was managed by a Madsen Orbiter 922 audiometer and the signal to noise ratio (SNR) was perfectly adjusted. 
*Cl users

For $\mathrm{Cl}$ users, tests were made in free field in an audiometric booth. In all cases only one ear was stimulated. When a subject was fitted with two implants, only the best ear was kept.

For NH subjects, the Fournier's lists and the cocktail-party noise were first processed by the $\mathrm{Cl}$ simulator and then passed through the audiometer and finally delivered on the right ear by a TDH 39 headset.

For the two groups of subjects the experiment consisted in a speech audiometry in noise. The maximum level delivered was below $65 \mathrm{~dB}$ SPL. According to the conditions requested by the ethics committee it did not exceed the $80 \mathrm{~dB}$ SPL limitation recommended for professional noises exposure.

The recognition scores of the $\mathrm{Cl}$ users were collected before and after cleaning the microphone, leading to two different situations ("dirty and clean"). Our experiment took place at the beginning of the periodical clinical check and device setting occurring periodically at the CRIC (Cochlear Implant Setting Center) located in the ORL department of the Edouard-Herriot University hospital of Lyon. This check-up consists of an appointment with a speech therapist, a setting of the implant parameters and a clinical examination. Consequently, in our study the microphone cleaning occurred before the classical check-up. This device check follow-up is carried out at least once a year.

We used two different procedures to clean the microphones ports depending on the type of speech processor: A "Brush and Blow" technique (commonly used in French hearing care centers) and the replacement of the protective filters. The "brush and blow" procedure consists in brushing and blowing a flow of dry air on the microphones ports to remove dirt and dust.

The following tasks were realized in our work:

-Verification of the patient's medical file,

-Short training session to help the patient to understand the instructions,

-First test with the Fournier's lists, before cleaning the microphone. The lists were presented to the patient with an increasing level of difficulty (SNR decreased from $18 \mathrm{~dB}$ to $-3 \mathrm{~dB}$; step $-3 \mathrm{~dB}$ ),

-Microphone cleaning,

-Second test with different Fourier's lists after cleaning the microphone.

The full session lasted about 30 minutes. Sixteen Fourier's lists were used in this experiment ( 8 before cleaning and 8 after) and the lists were not repeated.

\section{*NH subjects}

For $\mathrm{NH}$ subjects, stimuli were delivered to only one ear similar to the experiment with $\mathrm{Cl}$ users. Furthermore, we chose to test the subjects on the right ear considering that the left brain auditory cortex is specialized in processing speech. 
Eight conditions have been considered:

-2 coding schemes (CIS-like and n-of-m)

-4 degrees of microphone sensitivity loss.

Five levels of SNR were tested for each condition leading to $40(2 * 4 * 5)$ combinations. Each combination was assigned to a Fournier's list so that the lists were not repeated. Each session started with a short training period to help the listener to understand the instructions. Then the 40 lists were randomly presented to each subject. The session lasted about 45 minutes.

\subsection{Mathematical representation}

The recognition percentages versus the SNR can be represented by a sigmoid regression curve (figure 4).

Three features were considered here:

-the SNR corresponding to $50 \%$ of the maximum recognition plateau denoted here $\mathrm{x}_{50 \%}$;

-the "slope" (SNR interval, given in $\mathrm{dB}$, between $25 \%$ and $75 \%$ of the maximum recognition) and denoted here $\Delta_{75 \%-25 \%}$;

-the top asymptote $y_{\max }$ shows the maximum recognition score.

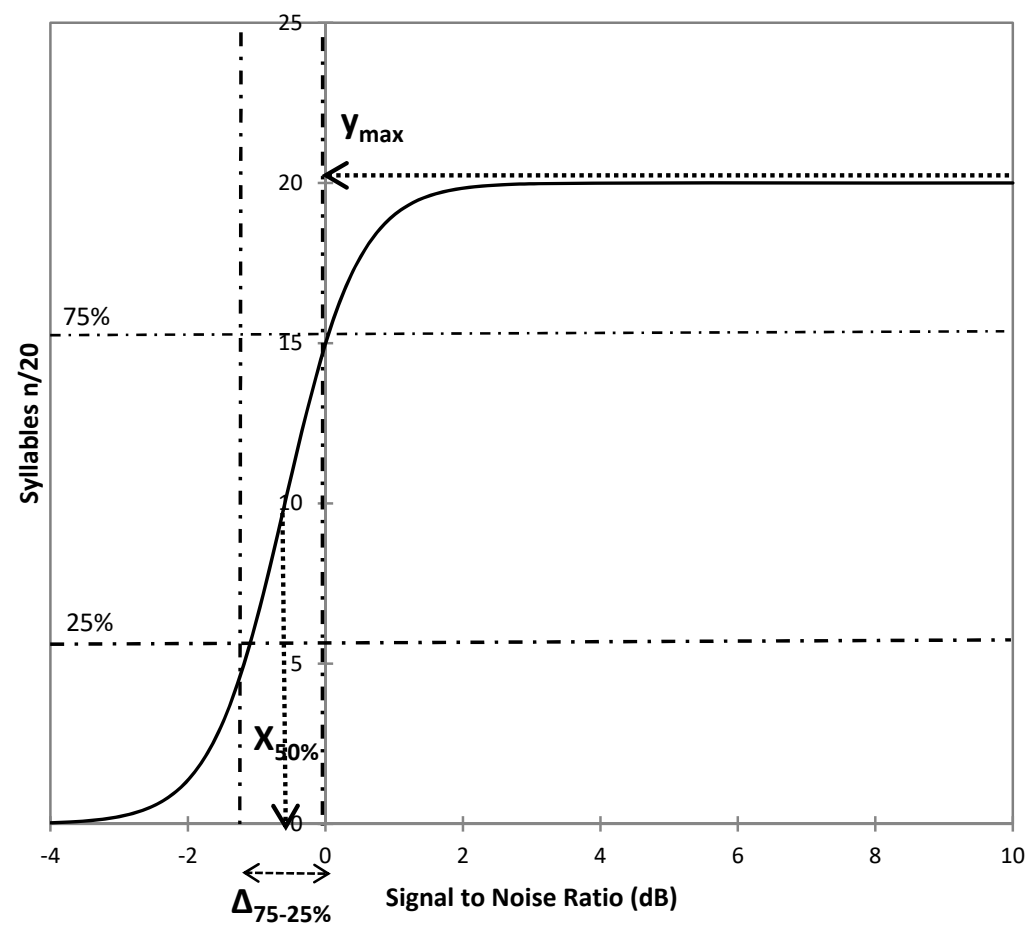

Figure 4: Fitting of the recognition percentages by a sigmoid curve 
These analytical values are represented on the sigmoid curve. The minimum recognition was $0 \%$ (seen for SNR $=-3 \mathrm{~dB}$ ). Thus, the sigmoid equation is:

$$
y=\frac{a}{1+e^{-b(x-c)}}
$$

Where:

$-y$ is the recognition percentage

$-x$ is the SNR

-a is $y_{\max }$

$-c$ is $X_{50 \%}$

-b is linked to the slope: $b=2.2 / \Delta_{75 \%-25 \%}=>\Delta_{75 \%-25 \%}=2.2 / \mathrm{b}$

\section{RESULTS}

\subsection{Percentage of intelligibility}

\subsection{1. $\mathrm{NH}$ subjects}

Figure 5 shows the percentage of correctly repeated syllables, function of the SNR, for the simulated strategies a) CIS-like b) $\mathrm{n}$-of-m. The intelligibility is represented for the 4 degrees of simulated soiled microphones (no attenuation, $800 \mathrm{~Hz}$ low pass filtering at $-2,-7$ and $-9 \mathrm{~dB}$ per decade)
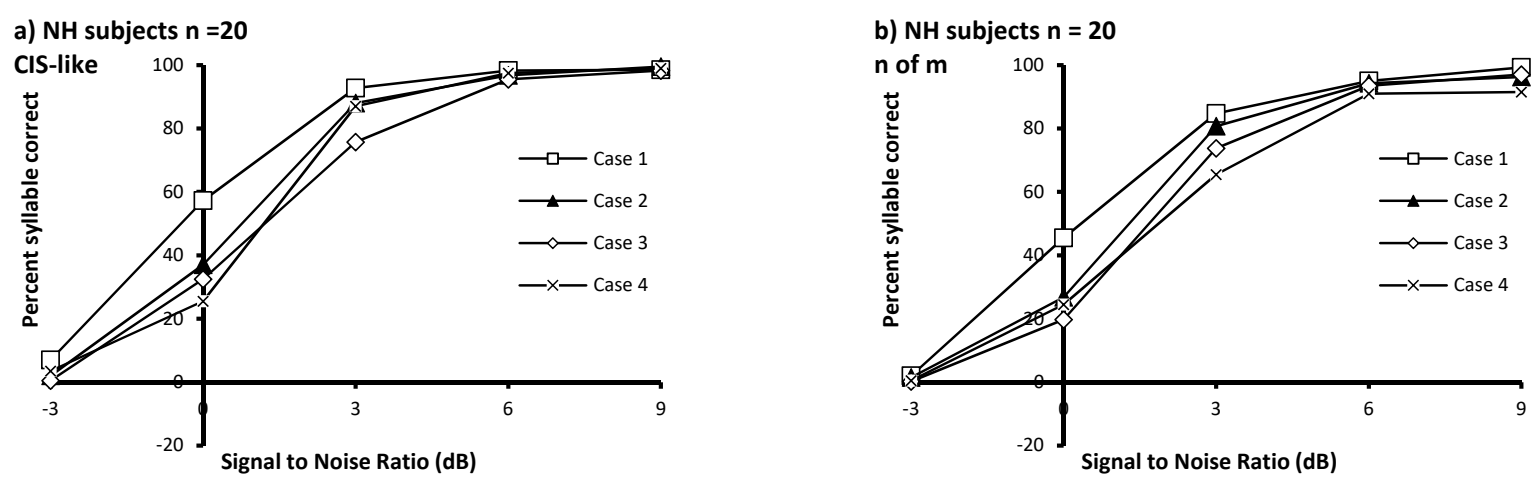

Figure 5: Intelligibility with respect to the SNR in four cases of simulated soiled microphones, a) for the CIS-like strategy, b) for the n-of-m strategy.

- $\quad N$-of-m coding scheme; ANOVA and post-hoc analyses

A two-factor repeated measure ANOVA was used to analyze the results. It indicated a significant effect of SNR $\left[p<10^{-4}\right]$ and of the degree of soiling $\left[p<10^{-4}\right]$. Furthermore a significant interaction was seen between them $\left[p<10^{-4}\right]$.

Table 1 shows the results of Wilcoxon's tests taken for the paired post-hoc comparisons, between soiling conditions, with respect to the SNR; significant differences are highlighted.

Largest differences were located at 9, 3 and $0 \mathrm{~dB}$ of SNR (Tab 1). The results indicated that Case 1 was better than Cases 2 and 4 at the highest SNR ( $9 \mathrm{~dB})$. For the SNR 3dB the intelligibility for 
the Case 1 was significantly higher than for the Cases 3 and 4 and also Case 2 was better than Case 4 . Finally, Case 1 was better than Cases 2, 3 and 4 for the SNR $0 \mathrm{~dB}$.

\begin{tabular}{lllllll}
\hline $\begin{array}{l}\text { NH n-of-m } \\
\text { p-value }\end{array}$ & C1-C2 & C1-C3 & C1-C4 & C2-C3 & C2-C4 & C3-C4 \\
\hline SNR9 & 0.0345 & 0.0982 & 0.0033 & 0.5556 & 0.2805 & 0.1654 \\
SNR6 & 0.2638 & 0.6714 & 0.0948 & 0.8834 & 0.1579 & 0.3092 \\
SNR3 & 0.5549 & 0.0067 & 0.0145 & 0.0725 & 0.0048 & 0.1900 \\
SNR0 & 0.0058 & 0.0004 & 0.0019 & 0.3367 & 0.6307 & 0.2575 \\
SNR-3 & 0.6675 & 0.0650 & 0.1048 & 0.1736 & 0.1736 & 0.7728 \\
\hline
\end{tabular}

Tab 1: $n$-of-m, paired comparisons between the 4 cases with respect to the SNR

- CIS-like coding scheme;ANOVA and post-hoc analyses

The two-factors repeated measures ANOVA indicated a significant effect of SNR $\left[p<10^{-4}\right]$ and of the degree of soiling $\left[p<10^{-4}\right]$. Furthermore a significant interaction was seen between them $[p<$ $10^{-4}$ ]. Table 2 shows the results of Wilcoxon's tests for the paired post-hoc comparisons with respect to the SNR. The significant differences are highlighted.

Largest differences are located between 3 and $-3 \mathrm{~dB}$ of SNR (Tab. 2). The results indicate that Case 1 was better than Cases 3 and 4 and that Case 2 was better than Case 3 at the SNR $3 \mathrm{~dB}$. For the SNR OdB the intelligibility for Case 1 was significantly higher than for the Cases 2, 3 and 4. Finally, Case 1 was better than Case 3 for the SNR -3dB.

\begin{tabular}{lcccccc}
\hline $\begin{array}{l}\text { NH ClS-like } \\
\text { p-value }\end{array}$ & C1-C2 & C1-C3 & C1-C4 & C2-C3 & C2-C4 & C3-C4 \\
\hline SNR9 & 0.2031 & 0.8514 & 0.5862 & 0.2837 & 0.3458 & 0.5160 \\
SNR6 & 0.2423 & 0.0688 & 0.7970 & 0.4087 & 0.4991 & 0.3044 \\
SNR3 & 0.1369 & 0.0006 & 0.0187 & 0.0088 & 0.5145 & 0.0238 \\
SNR0 & 0.0035 & 0.0028 & 0.0006 & 0.4973 & 0.0925 & 0.2754 \\
SNR-3 & 0.0649 & 0.0080 & 0.2770 & 0.2342 & 0.5067 & 0.1003 \\
\hline
\end{tabular}

Tab 2: CIS-like, intelligibility paired comparisons between the 4 cases with respect to the SNR

Strategies are compared on figure 6. 

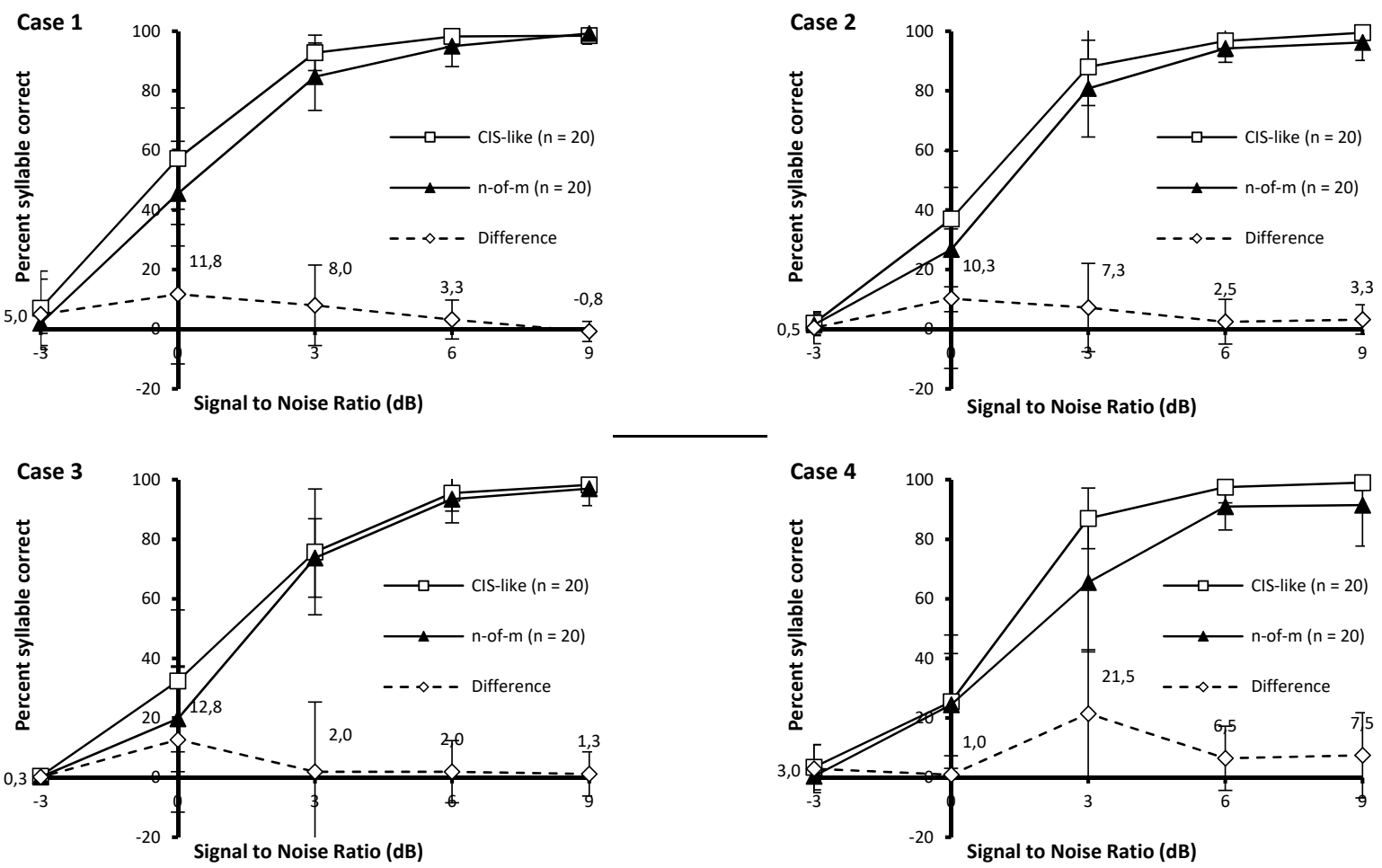

Figure 6: Intelligibility with respect to the SNR in four cases of simulated soiled microphones, comparison of CIS-like and n-of-m.

\subsection{2. $\mathrm{Cl}$ users}

The effect of cleaning the microphone ("Brush and Blow" procedure) was evaluated on the recognition percentages; it is represented on figure 7 according to the strategies. It indicates the efficiency of cleaning. The heterogeneity in the results did not allow definitive conclusions and only indicated clues.

\section{- $\quad N$-of-m coding schemes; ANOVA analysis}

For the n-of-m strategies the improvements (figure $7 \mathrm{~b}$ ) were mostly noticed for the high values of the SNR $(12,15$ and $18 \mathrm{~dB})$ and there was an average improvement of $2.7 \%$ in syllable recognition after cleaning (corresponding to " $1 / 2$ syllable" per list).

The two-factors repeated measures ANOVA indicated a significant effect of SNR $\left[p<10^{-4}\right]$ but no difference between before and after cleaning [ $p=0.056]$.

\section{- CIS-like coding schemes; ANOVA analysis}

For the CIS-like strategy (figure 7a), the best improvements were observed in the SNR range 0 to $9 \mathrm{~dB}(0,3,6$, and $9 \mathrm{~dB})$ and the average improvement after cleaning was $2.9 \%$.

The two-factors repeated measures ANOVA indicated a significant effect of SNR $\left[p<10^{-4}\right]$ but no difference between before and after cleaning $[p=0.12]$. 
a) $\mathrm{Cl}$ users, CIS-like $\mathbf{n}=19$

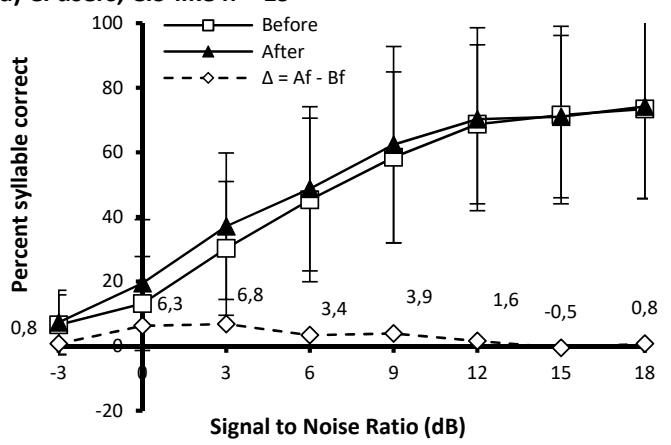

b) $\mathrm{Cl}$ users, $\mathrm{n}$-of-m $\mathrm{n}=\mathbf{4 2}$

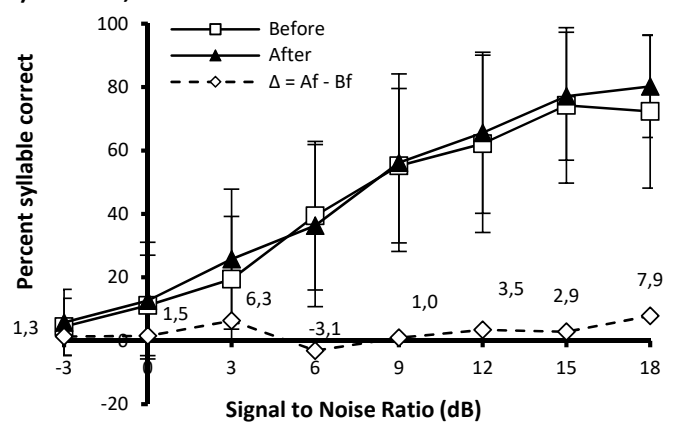

Figure 7: Intelligibility with respect to the SNR before and after cleaning the microphones' port ("Brush and Blow" cleaning procedure), a) CIS-like strategies, b) n-of-m strategies

\section{$\underline{\text { Protective filter replacement; ANOVA analysis }}$}

In the case of the replacement of the protective filter, there was an average improvement of $4.7 \%$ in syllable recognition after replacement (corresponding to one syllable per list); the results are represented on figure 8.

The two-factors repeated measures ANOVA indicated a significant effect of SNR $\left[p<10^{-4}\right]$ but no difference was seen between before and after cleaning $[p=0.43]$ and no interaction occurred between the two factors $[p=0.91]$
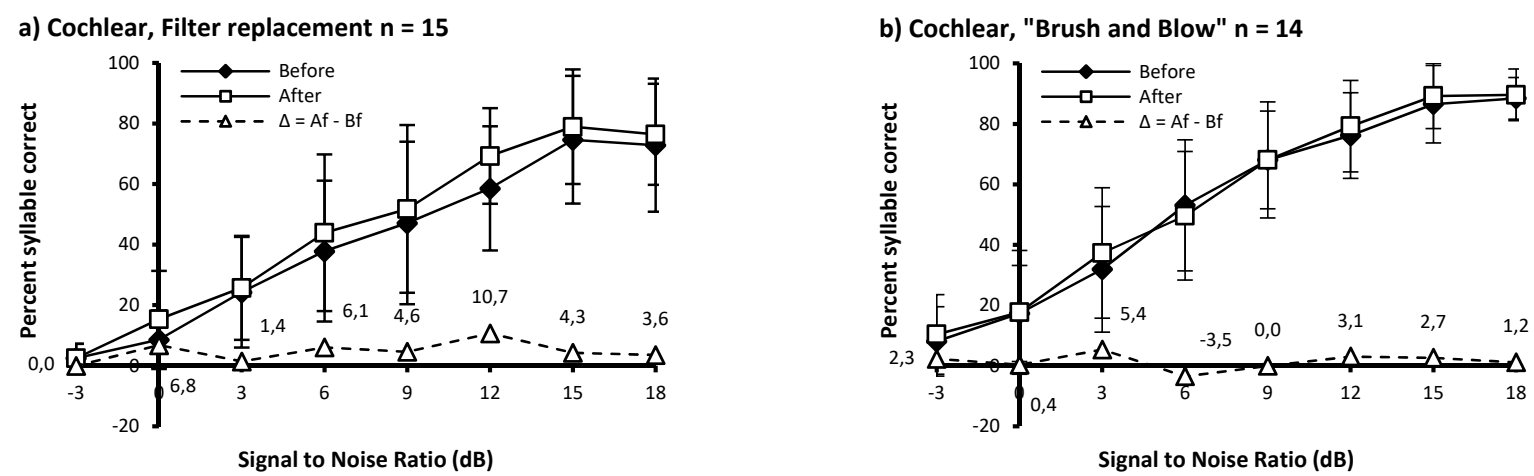

Figure 8: Intelligibility with respect to the SNR before and after cleaning the microphones' port, a) Cochlear processors, protective filters replacement, b)Cochlear processors, "Brush and Blow" cleaning procedure 


\subsection{Audiological values}

\subsection{1. $\mathrm{NH}$ subjects}

- $\quad \mathrm{N}$-of-m coding schemes; ANOVA and post-hoc analyses

A one-factor repeated measures ANOVA indicated a significant effect of the soiling degree on $x_{50 \%}\left[p<10^{-4}\right]$ but did not reveal a difference for the features $\Delta_{75-25 \%}[p=0.52]$ and $y_{\max }[p=0.32]$.

We can see on tab 3 that the feature $x_{50 \%}$ was better for Case 1 compared to Cases 2, 3, 4. Also, $\mathrm{x}_{50 \%}$ for Case 2 was significantly better than for Case 4 .

\begin{tabular}{lllllll}
\hline $\begin{array}{l}\text { NH n-of-m } \\
\text { p-value }\end{array}$ & C1-C2 & C1-C3 & C1-C4 & C2-C3 & C2-C4 & C3-C4 \\
\hline $\mathrm{x}_{50 \%}$ & 0.0076 & 0.0003 & 0.0008 & 0.1403 & 0.0458 & 0.3411 \\
\hline
\end{tabular}

Tab 3: CIS-like, feature $x_{50 \%}$ paired comparisons between the 4 cases

- CIS-like coding scheme; ANOVA and post-hoc analyses

Again we used a one-factor repeated measure ANOVA.It indicated a significant effect of the soiling degree on the feature $x_{50 \%}\left[p<10^{-4}\right]$ but did not reveal a difference for the features $\Delta_{75-25 \%}[p=$ $0.48]$ and $y_{\max }[p=0.43]$.

We can see on tab 4 that the feature $x_{50 \%}$ was better for Case 1 compared to Cases 2, 3, 4. Also, $\mathrm{x}_{50 \%}$ for Case 2 was significantly better than for Case 3 .

\begin{tabular}{ccccccc}
\hline $\begin{array}{c}\text { NH CIS-like } \\
\text { p-value }\end{array}$ & C1-C2 & C1-C3 & C1-C4 & C2-C3 & C2-C4 & C3-C4 \\
\hline $\mathrm{X}_{50 \%}$ & 0.0038 & 0.0002 & 0.0012 & 0.0349 & 0.1978 & 0.1851 \\
\hline \multicolumn{7}{l}{ Tab 4: ClS-like, feature $\mathrm{x}_{50 \%}$ paired comparisons between the 4 cases }
\end{tabular}

\subsubsection{Cl users}

- $\quad n$-of-m coding schemes; Wilcoxon's tests

The Wilcoxon's tests indicated no significant effect of the cleaning on the curve features $x_{50 \%}$ $[p=0.66], \Delta_{75-25 \%}[p=0.85]$ and $y_{\max }[p=0.35]$.

- CIS-like coding schemes; Wilcoxon's tests

The Wilcoxon's tests indicated no significant effect of the cleaning on $x_{50 \%}[p=0.63], \Delta_{75-25 \%}[p$ $=0.29]$ and $y_{\max }[p=0.41]$.

\subsubsection{Filter replacement procedure}

- Wilcoxon's tests for the procedure "filter replacement"

The Wilcoxon's tests indicated no significant effect of the cleaning on the curve features $\mathrm{x}_{50 \%}$ $[p=0.96], \Delta_{75-25 \%}[p=0.62]$ and $y_{\max }[p=0.19]$. 


\section{GENERAL COMMENTS}

\section{About the present study}

Several indications came out of this work:

- The higher the SNR, the better the recognition (this is obvious). What is less obvious is the SNR for the ceiling effect. For NH subjects it was $6 \mathrm{~dB}$ for $\mathrm{ClS}$ and $\mathrm{n}$-of-m whatever the microphone drift. Also, the floor effect ( $0 \%$ recognition) was always reached at $-3 \mathrm{~dB}$ of SNR. For $\mathrm{Cl}$ users, the ceiling was seen for $15 \mathrm{~dB}$, before and after cleaning for $\mathrm{CIS}$ and $\mathrm{n}$-of-m.

- Cleaning improves the recognition (this is sensible). For NH subjects the improvement could not be seen for the extreme values of the SNR range $(-3 d B$ and $+9 d B)$. The importance of cleaning was maximum for the medium values of the SNR range $(0$ and $3 \mathrm{~dB})$. For $\mathrm{Cl}$ users, the results were similar to the $\mathrm{NH}$ subjects; only the improvement was reduced.

- Considering the sound coding strategies, the recognition percentages obtained with CIS are better than those seen with $n$-of-m, except for the extreme values (floor and ceiling effects).

Some exceptions can be pointed out:

For NH subjects, ceilings were not the same for $\mathrm{ClS}$ and n-of-m, when the microphone was strongly dirty. For $\mathrm{Cl}$ users when the microphone was clean, the $\mathrm{n}$-of-m scheme led to better results than CIS at the high values of the SNR. May be due to the heterogeneity of $\mathrm{Cl}$ users, this result was not significant.

Consequently, the robustness of this finding has to be established and further studies should be done to clarify this aspect. So far we may suggest that the coding scheme could be adapted to the noisy environment. Also, a cleaning protocol should be inserted in the regular check of the machines.

Another aspect which was seen was the cleaning procedure: "Brush and Blow" compared to the microphone protective filter replacement.

Basically it seems that the replacement led to a better improvement of the recognition percentage than the "Brush and Blow" procedure. In our experiment, the "Brush and Blow" procedure was performed by a qualified audiologist; it may be different when audiologists which are not used to this procedure have to accomplish this task, mostly if they are worried about damaging the device. If the filter replacement is cheap and fast, it may be a safer approach to systematically change the head filter protecting the microphone, but it has to be seen in our work that it is not worthy to be done.

Going further, a replacement of the microphone can be suggested but other questions are open by this procedure. Obviously, a new microphone would be the best solution, but this is more costly and the connection can lead to contact problems; the junction can be fragile and could be a source of hard failure. Also, for reasonable and good values of the SNR, the difference of performances seen between a new microphone and a microphone with a small drift (50\% of the users) is very light and not significant (result seen with the NH subjects). Also, cleaning the microphone in the case of CIS users, did not lead to a dramatic increase of the performances (possibly, the microphones were only slightly soiled). 


\section{About the microphone}

Aging occurs for all components but the action is not the same for all. A breakdown is an acute failure, and it will be followed by an immediate repair. When the fault is irregular, it is more difficult to stop it, because no one knows when the incident will occur and this is a very worrying situation. When the trouble comes insidiously, it is often not perceived and the subject loses progressively his efficiency without being aware of it. This is more or less what happens during the life; someone gets older, in good health, and he hardly notices the decrease of this aptitude to perform many tasks in the life, mentally or physically. Slopes get steeper, mental activities get harder, but the subject can still cope, even if it is a little bit more difficult. This is typically what happens with the microphone; it does not break, but it gets less efficient and the user is not aware of it. This is why this evolution must be studied; what are the consequences and how we can beat it with rather inexpensive actions, in time and in money? The community surrounding a patient, a cochlear-implantee in our case, must be aware of this condition and should take the necessary steps to restore the good conditions attached to the machine. Several protective measures are easy to imagine. To get a good protection to moisture, grease, sweat, dust, debris, a head filter is a good shield for the microphone and it should be systematically checked by the audiologist each time the patient come for a follow up with the medical team. The degree of sensitivity reduction has its importance; this reduction can be beaten; we should lower the signal perturbation, with an attenuation and a systematic shift of the frequencies seen with an increase of the stiffness and of the dirt of the membrane [7].

A study, in simulation, can bring situations which are not often seen in real life; it emphasizes effects which are attached to extreme conditions and sheds more light on the issue we want to study. The influence of the modifications of the properties of the microphone membrane was tested in classical hearing aids checked by a hearing aid analyzer [16]. Then all the situations (coding scheme, microphone drift, signal to noise ratio) were presented to the same $\mathrm{NH}$ subjects, a rather homogenous group, and powerful paired student's tests could be conducted. Obviously, the study in simulation cannot ignore the behavior and the performances obtained with $\mathrm{Cl}$ users as this group is the target for this technology.

\section{About the listening conditions}

Three approaches have been done in this work, simulation with NH subjects, simulation of the microphone drift and addition of noise. Noise needs to be introduced in this kind of work for two reasons. First noisy situations are very common in the usual environment, for $\mathrm{Cl}$ users and for $\mathrm{NH}$ subjects. Secondly, to get understandable results, one must avoid the floor and the ceiling effects; the range of the noise, associated with a good choice of the speech signal, allows to fulfill this requirement. Experimental conditions are a real issue, and their choice is far from being easy; pilot studies are more than necessary [17].

Then, the assessment of a listening condition cannot be done only by the comparison of recognition percentages. Other speech tests are worthy to be considered as they throw another glance on the performance. These parameters help to understand a scatter plot, and we have calculated the SNR for the mean recognition, the slope representing the action of the noise on the percentages, and the ceiling indicating the maximum performance reached in a given situation. In order to do so, a mathematical fitting was necessary, and finally it was worth to be done regarding the data synthesis it provided. 
Practically, it is necessary for hearing aid management and rehabilitation of $\mathrm{Cl}$ recipients that their devices are functioning well and are set by an audiologist during the check which is periodically done in all the clinics. The decrease of the microphone sensitivity is part of this clinical evaluation and it should be corrected, as much as possible. Nevertheless the correlation between the decline of the microphone sensitivity and its consequences on speech recognition scores was not found to be dramatic.

\section{Strategy}

As indicated above, CIS strategy was less sensitive to the microphone drift than the n-of-m coding, mostly in the middle of the SNR range. This is an indication to the audiologists in charge of $\mathrm{Cl}$ settings and to the manufacturers.

Is it only due to the stimulation speed, the spacing between the electrodes which lowers the overlapping? Is the spectrum coverage more useful to the brain than a selection of the phonetic features presented to the brain? For many researchers, Cls work, "because there is a brain behind" and we should not forget the efficiency of this premier organ of our body. We give information to the brain, then let it work.

Then other settings on the strategy have their influence: sampling rate, filters settings, length of the time frame (window), and they have been studied by many authors [1, 18, 19, 20]. Most of the studies concluded to an advantage of the CIS coding over n-of-m. Then, the Advanced Combination Encoder (ACE) scheme is very efficient; it uses a very fast updating of the data and therefore provides a fine temporal analysis of the signal, indicating that spectrum is not the only component of speech.

Then the results are subjected to the patient's condition and mostly to the degree of nerve survival and to the intellectual background; it has consequences on the appropriate strategy for individual implant patient [21]. A good adjustment of a strategy to a subject is a goal to be reached, and it should be adapted throughout the time.

\section{Noise}

The robustness of the strategies should be also seen in noise. In a background noise, the performance of $\mathrm{Cl}$ users is significantly better when using a full filter bank approach ("CIS-like") in comparison to using a peak picking method. Consequently, when the signal is distorted (noise or microphone drift) CIS coding seems to be more robust, but this is not unanimously accepted [22].

With $\mathrm{Cl}$ users the performance in noise is very difficult to interpret and it is related to the patient's characteristics and to the wide variability in this group of people. The degree of deterioration in noise was similar with each strategy.

Also the number of channels needed to understand speech in noise is still an open question [23]. More channels are needed to understand speech in noise than in quiet, both for $\mathrm{Cl}$ users and $\mathrm{NH}$ listeners, even if results are better in simulation with $\mathrm{NH}$ subjects. Again, the number of electrodes (channels) must be kept low to reduce the overlap between the electrodes.

A recommendation which is classically delivered when hard of hearing people are concerned is to provide good listening conditions (high SNR) and to speak slowly and distinctively. This situation is not always easy to meet and we should reduce the noise level as much as we can. Consequently 
many studies have been done to lower the noise, in other words to increase the SNR [24, 25, 26]. This is attractive, but not always easy to implement as hypotheses should be made about the noise.

Aging is an important issue for human, animal, vegetal and machines. "Time flows and we follow". Wisdom is to adapt and to find the best strategies to reduce the consequences; this is also true for the cochlear implants microphones and that was the aim of this dissertation: how can we lower the impact of time?

According to us, to lower that impact on the microphones several suggestions can be made to keep the microphone function:

- "Stop the time flow" (unfortunately this is not possible),

- Use the Brush a Blow cleaning procedure,

- Make a head filter replacement,

- Perform a microphone replacement.

The cheapest is to apply a "Bruch and Blow" cleaning procedure at every routine check and the results on the performance should be satisfactory. So we can recommend this procedure to the specialized teams in charge of the $\mathrm{Cl}$ patient's care.

\section{CONCLUSION}

In this work we have studied the effect of soiling and aging of the microphones of cochlear implants on the recognition of syllables in the French Fournier's disyllabic lists of words.

Several parameters were studied in this work:

- The influence of the coding strategies (CIS-like and n-of-m),

- The action of the signal to noise ratio,

- The effect of cleaning the microphones.

Experiments were conducted with $\mathrm{Cl}$ users and $\mathrm{NH}$ listeners as the two approaches are complementary. Also, two procedures to clean the microphones were considered, the classical "Brush and Blow" and the replacement of the protective filter of the microphone. With $\mathrm{Cl}$ users we were in the true situation, and with $\mathrm{NH}$ subjects we could see more analytically the issue and we could perform a more precise analyses using data taken on the same person.

Results indicated that:

- The CIS-like coding strategies were more robust than the $\mathrm{n}$-of-m strategies regarding the microphone drift; the difference could reach two syllables in a list $(10 \%)$ and it was seen mainly in the middle of the SNR range,

- The SNR effect was very important, the recognition percentages ranging from 0\% (SNR = $3 \mathrm{~dB}$ ) to $100 \%$ (SNR $=9 \mathrm{~dB}$ or higher). Results were generally lower with $\mathrm{Cl}$ users than with $\mathrm{NH}$ subjects. In some circumstances, the difference could be very small,

- Cleaning the microphones improved the percentages, mostly in the case of NH subjects in simulation as more extreme situations could be considered and they were tested on the same subjects (through paired comparisons). 
- With $\mathrm{Cl}$ users, it was difficult to get significant results, probably due to the heterogeneity of the group, and this is very general when pathology is considered. Also, the protective filter replacement seemed to be more efficient than the classical "Brush and Blow" cleaning procedure, but this effect was not seen everywhere on the SNR range, and this result was not statistically proven.

Finally, we recommend the use of the CIS-like strategies over the n-of-m coding strategies whenever it is possible. Also, in the regular checkup which is programmed in the follow-up of $\mathrm{Cl}$ users, we suggest that the audiologists perform a cleaning of the microphone (the "Brush and Blow" procedure is not aggressive) as the drift is very insidious and often not noticed by the subjects. In some cases (very dirty microphones) it would be helpful for the patient.

We cannot stop aging, but we can attenuate its effects and it was the aim of this work on the microphone drift for cochlear implants ("a pin hole in a wide issue").

\section{ACKNOWLEDGMENTS}

The authors are grateful to the persons and the bodies who participated to the study; $M$. Kevin Perreaut who initiated the work, Dr Fabien Seldran and Dr Fabien Millioz for the scientific contribution and Ms. Evelyne Veuillet for the links with the ethic committee. We also wanted to thank the members of the CRIC Lyon and the staff of the Edouard-Herriot hospital for their collaboration, the subjects who listened to the Fourier's lists, the Hospitals of Lyon and the Polytechnic School of the University of Lyon.

\section{REFERENCES}

1. Aguiar DE, Taylor NE, Li J, Gazanfari DK, Talavage TM, Laflen JB, et al. Information theoretic evaluation of a noiseband-based cochlear implant simulator. Hear. Res. 2015;

2. Battmer R-D, O'Donoghue GM, Lenarz T. A multicenter study of device failure in European cochlear implant centers. Ear Hear. 2007;28:95S-99S.

3. Balkany TJ, Hodges AV, Buchman CA, Luxford WM, Pillsbury CH, Roland PS, et al. Cochlear implant soft failures consensus development conference statement. Cochlear Implants Int. 2005;6:105-22.

4. Schweitzer C. Mind the ports! The Effect of Severe Microphone Inlet Occlusion: port disasters, or how everyday activities can lead to severe microphone occlusion in a directional hearing aid. Hear. Rev. 2008;15:14.

5. Pereira AM, Melo TM de, Pereira AM, Melo TM de. Repair issues associated with cochlear implants external components: the influence of age and time of use. Rev. CEFAC. 2014;16:1419-25.

6. Silverman CA, Linstrom CJ, Gilston N, Schoepflin JR. Repair Issues Associated with Cochlear Implants. Cochlear Implants Int. 2010;11:469-72.

7. Razza S, Burdo S, Bonaretti S. Acoustical signal check: microphone integrity evaluation through a common hearing aid analyzer. 5th Object. Meas. Symp. Cochlear Implants ABI. 2007. p. 19-22.

8. Razza S, Burdo S. An underestimated issue: unsuspected decrease of sound processor microphone sensitivity, technical, and clinical evaluation. Cochlear Implants Int. 2011;12:114-23. 
9. Wouters J, McDermott HJ, Francart T. Sound Coding in Cochlear Implants: From electric pulses to hearing. IEEE Signal Process. Mag. 2015;32:67-80.

10. Wilson BS, Lawson DT, Zerbi M, Finley CC, Wolford RD. New processing strategies in cochlear implantation. Am. J. Otol. 1995;16:669-75.

11. Garnham C, O'Driscoll M, Ramsden And R, Saeed S. Speech understanding in noise with a Med-El COMBI 40+ cochlear implant using reduced channel sets. Ear Hear. 2002;23:540-52.

12. Dorman MF, Loizou PC, Fitzke J, Tu Z. Recognition of monosyllabic words by cochlear implant patients and by normal-hearing subjects listening to words processed through cochlear implant signal processing strategies. Ann. Otol. Rhinol. Laryngol. Suppl. 2000;185:64-6.

13. Shannon RV, Fu Q-J, Galvin J. The number of spectral channels required for speech recognition depends on the difficulty of the listening situation. Acta Oto-Laryngol. Suppl. 2004;50-4.

14. Dorman MF, Loizou PC. The identification of consonants and vowels by cochlear implant patients using a 6-channel continuous interleaved sampling processor and by normal-hearing subjects using simulations of processors with two to nine channels. Ear Hear. 1998;19:162-6.

15. Traunmüller H. Analytical expressions for the tonotopic sensory scale. J. Acoust. Soc. Am. 1990;88:97-100.

16. Serra P-O. Effet de l'entretien des aides auditives sur leurs performances [Audiology diploma dissertation]. University of Montpellier; 2015.

17. K.Perreaut, S.Gallego, C.Berger-Vachon, F.Millioz. Influence of Microphone Encrusting on the Efficiency of Cochlear Implants Preliminary Study with a Simulation of CIS and " $n-o f-m$ " Strategies. AMSE Journals- Modelling C. 2014; 75-2: 199-208.

18. Kerber S, Seeber BU. Sound localization in noise by normal-hearing listeners and cochlear implant users. Ear Hear. 2012;33:445-57.

19. Hazrati O, Loizou PC. Comparison of two channel selection criteria for noise suppression in cochlear implants. J. Acoust. Soc. Am. 2013;133:1615-24.

20. Shannon RV, Zeng FG, Kamath V, Wygonski J, Ekelid M. Speech recognition with primarily temporal cues. Science. 1995;270:303-4.

21. Wilson BS, Finley CC, Farmer JC, Lawson DT, Weber BA, Wolford RD, et al. Comparative studies of speech processing strategies for cochlear implants. The Laryngoscope. 1988;98:1069-77.

22. Nelson PB, Jin S-H, Carney AE, Nelson DA. Understanding speech in modulated interference: Cochlear implant users and normal-hearing listeners. J. Acoust. Soc. Am. 2003;113:961-8.

23. Loizou PC, Dorman MF, Tu Z, Fitzke J. Recognition of sentences in noise by normal-hearing listeners using simulations of speak-type cochlear implant signal processors. Ann. Otol. Rhinol. Laryngol. Suppl. 2000;185:67-8.

24. Jeanvoine A, Gnansia D, Truy E, Berger-Vachon C. Contribution of Noise Reduction Algorithms: Perception Versus Localization Simulation in the Case of Binaural Cochlear Implant (BCl) Coding. Emerg. Trends Comput. Biol. Bioinforma. Syst. Biol. 2015;307-24. 
25. Kallel F, Laboissiere R, Ben Hamida A, Berger-Vachon C. Influence of a shift in frequency distribution and analysis rate on phoneme intelligibility in noisy environments for simulated bilateral cochlear implants. Appl. Acoust. 2013;74:10-7.

26. Hu Y, Loizou PC. A new sound coding strategy for suppressing noise in cochlear implants. J. Acoust. Soc. Am. 2008;124:498-509. 